\title{
A Tale of Giants Stealing from Dwarfs
}

\author{
Duncan A. Forbes \\ Centre for Astrophysics \& Supercomputing, Swinburne University, \\ Hawthorn VIC 3122, Australia
}

\section{Disrupting Dwarf}

Cold Dark Matter simulations predict 10-100 $\times$ more dwarf satellite galaxies than are observed. Some of these 'missing satellites' may have been accreted, along with their globular clusters (GCs), by giant galaxies (Cote et al. 1998). But examples of dwarfs in the early stages of disruption have remained elusive.

However images of the Tadpole galaxy by the ACS on HST contained a background spiral with a candidate 'disrupting dwarf' (ie two tails were seen coming from the dwarf). A 1 hr Keck spectrum of the spiral $(\mathrm{V}=18)$ and dwarf $(\mathrm{V}=23)$ confirmed the physical association and gave a redshift of 0.15 . We fit Sersic profiles to the surface brightness and found the dwarf has properties similar to a dE galaxy with blue central colors (Forbes et al. 2003).

Comparison with simulations suggests the original dwarf had a disk, ie a dIrr galaxy (Mayer et al. 2001). As it orbits, tails of stars are pulled off by tidal forces and the galaxy is accreted due to dynamical friction. As the starburst fades and it continues to lose stars it may resemble a $\mathrm{dSph}$ galaxy. The disrupted stars eventually add a metal-poor population to the giant galaxy halo. We would also expect the dwarf to contain some 25 GCs but these would have $\mathrm{V}>29$ and hence too faint to be seen in the ACS image. This observation illustrates the transformation of a dIrr to $\mathrm{dE}$ and possibly $\mathrm{dSph}$ via tidal stripping, and may help explain the dwarf galaxy morphology-density relation. Dwarfs can be transformed from one type into another if they get too close to a giant.

\section{Blues}

In the last few years it has become clear that the mean color of the red (metalrich) GCs in giant galaxies is strongly correlated with host galaxy mass (eg Forbes \& Forte 2001; Larsen et al. 2001). Strader, Brodie \& Forbes (2004) using high quality data, extended to include dwarf galaxies, have found a $6 \sigma$ correlation for blue (metal-poor) GCs of the type $\mathrm{Z} \sim \mathrm{L}^{0.15}$ (the slope is about half that for the red GCs). This implies that, like the red GCs, the blue GCs know about the potential they live in. Dwarf galaxies' GCs have a mean V-I of $\sim 0.88$ whereas giants have $\mathrm{V}-\mathrm{I} \sim 0.94$. This places strong limits on the number of accreted dwarf galaxy GCs that can be part of the blue GC subpopulation in giant galaxies. Dwarfs make up only a small part of the diet of giants. 


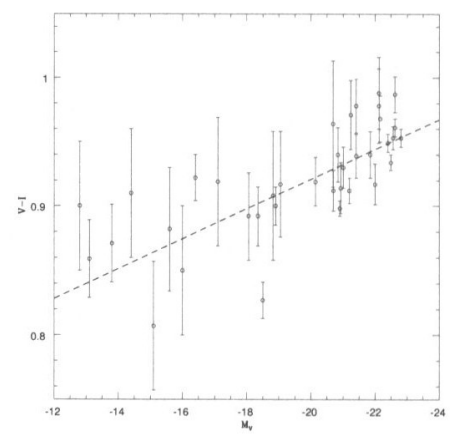

Figure 1. Mean V-I color for the blue (metal-poor) GC systems of galaxies versus the host galaxy luminosity. A $6 \sigma$ correlation is seen. Giant galaxies' GC systems can not simply be the accretion of many dwarf galaxy GC systems.

\section{Tidal stripping of GCs}

Another potential source of GCs for giant galaxies is to acquire them, via tidal stripping, from nearby galaxies. In this case, GCs, and possibly some field stars, are acquired, but in general the donor galaxy remains as a separate entity. Perhaps the best case for tidal stripping is that of NGC 1399 and NGC 1404 in the Fornax cluster. There is amble evidence for an ongoing interaction between the two galaxies and NGC 1404 reveals a low specific frequency of $\mathrm{S}_{N} \sim 2$ (eg Forbes, Brodie \& Grillmair 1997). Building on the ground-breaking work of Muccio and collaborators in the 1970s, Bekki et al. (2003) have modeled this process. Starting with an $\mathrm{S}_{N}=5$, Bekki et al. could reproduce the value of 2 by tidal stripping. They found an extended GC system and an eccentric orbit for NGC 1404 was required. The kinematics of the stripped GCs can, in principle, be used to probe the cluster-wide potential. A trend for $\mathrm{S}_{N}$ to increase with cluster-centric distance is predicted and mildly supported by current data (Forbes, Brodie \& Grillmair 1997). Giants steal GCs from dwarfs and sometimes eat the dwarf too (but most of the time the dwarf gets away).

\section{References}

Bekki, K., Forbes, D., Beasley, M., Couch, W., 2003, MNRAS, 344, 1334

Cote, P., Marzke, R., \& West, M., 1998, ApJ, 501, 554

Forbes, D., Brodie, J., Grillmair, C., 1997, AJ, 113, 1652

Forbes, D., Forte, J., 2001, MNRAS, 322, 257

Forbes, D., Beasley, M., Bekki, K., Brodie, J., Strader, J., 2003, Science, 301, 5637

Larsen, S., Brodie, J., Huchra, J., Forbes, D., Grillmair, C., 2001, AJ, 121, 2974 Mayer, L., et al. 2001, ApJ, 559, 754

Strader, J., Brodie, J., Forbes, D. 2004, AJ, 127, 3394 